\title{
Artemisa: An eco-driving assistant for Android Os
}

\author{
V.Corcoba Magaña \\ Dpto. de Ingeniería Telemática \\ Universidad Carlos III \\ Leganes, Spain \\ vcorcoba@it.uc3m.es
}

\author{
M. Muñoz-Organero \\ Dpto. de Ingeniería Telemática \\ Universidad Carlos III \\ Leganes, Spain \\ mmunoz@it.uc3m.es
}

\begin{abstract}
This paper proposes an eco-driving assistant that facilitates the user to learn the techniques of efficient driving. The Artemisa's assistant evaluates the driver's driving style taking into account some environmental as well as some vehicles's variables such as speed, gear, R.P.M, etc. Besides, tips are inferred to teach efficient driving habits. Compared to other similar systems, the Artemisa's assistant runs on a mobile device with Android O.S. and it does not need to install additional hardware.
\end{abstract}

Keywords-component; eco-driving; automotive; Android; expert system; Driving pattern factors

\section{INTRODUCTION}

VEHICLES are important energy consumers and major emitters of pollutant gases. The pollution they produce causes more deaths than road traffic accidents [1] [2]. Reducing energy consumption is a priority for governments and vehicle manufacturers.

Manufacturers can take actions to save energy (engines with lower consumption, weight reduction and aerodynamics improvement of the vehicle) and governments can make laws to ensure that manufacturers cannot produce cars contaminants, but this is not enough. To reduce energy consumption and greenhouse gas emissions is also necessary that the driver takes an efficient driving style. Adopting an efficient driving style can make savings of 10 to $25 \%$ of fuel [3] [4] [5] and also has other advantages (it improves comfort, reduces the risk and severity of accidents and there is less erode of vehicle components).

The Artemisa's eco-driving assistant presented in this paper aims to help the driver to take an efficient driving style. We evaluated the driver's driving style and, according to this evaluation results, eco-driving tips are displayed.

There are many proposals with the same purpose as Artemisa [6] [7], but they have several drawbacks:

- The assessment of the driver's driving style is not accurate because it does not consider the environmental variables such as the state of the road or the weather conditions.

- They require expensive additional hardware installation.

- They are not standard. Can only be applied to a particular model of vehicles.
The solution that we propose is based on the use of a mobile device running the Android O.S. [10] that many users already have and a Bluetooth module which costs around $\$ 50$. An Android smartphone, thanks to its large number of sensors, such as GPS and Internet access (3G/LTE), can obtain much information about the environment making it suitable as a data acquisition system.

For the phase of evaluation and generation of eco-driving tips we use an expert system that is executed on the mobile device. Android based mobile devices have enough power to perform complex tasks as we will see in the section describing the expert system.

Thanks to the use of the features offered by the Android smartphone, the Artemisa's eco-driving assistant is cheaper and more accurate than other solutions.

\section{STATE OF ART}

Eco-driving is a concept that has gained great importance in recent years. These driving techniques allow saving fuel independently of the vehicle's embedded technology, only depending on the driver's driving decisions.

The driver has to apply rules such as drive smoothly, accelerate gently and monitor the road ahead to avoid unnecessary braking. These rules do not require specific on board technological support but there are some issues that have to be considered and addressed:

- The driver needs to know the rules of efficient driving.

- It is difficult to change driving habits

- The driver requires a continuous motivation

- Driving is a very complex task in which there are multiple goals that may come into conflict with each other. For example, if the driver wants to arrive early at a destination, he or she will increase speed causing a conflict with the goal of saving fuel

To solve these issues we can use an eco-driving assistant which allows drivers to learn the rules of driving efficiently without having to attend classes and at the same time motivates them to apply them. 
There are several studies like Boriboonsomsin[11] that evaluates the suitability of eco-driving assistants to make the users acquire a more efficient driving style.

The use of efficient driving techniques has a positive impact on fuel saving as has been demonstrated in many studies such as Mierlo [12]

There are also several proposals that analyze which variables affect fuel consumption. Kuhler [13] introduced a set of ten variables. These variables are used in laboratories for consumption and emissions of vehicle fuel. Other authors such as André [14] and Fomunung [15] increased the number of parameters or replaced some of them to improve results. The drawback of these proposals is that they do not take into account the environment where the vehicle circulates that often has a significant influence on energy consumption.

\section{ARTEMISA'S ECO-DRIVING ASSISTANT}

The Artemisa's eco-driving assistant has two modules: the data acquisition system and the expert system. The data acquisition module is responsible for obtaining all the necessary variables to model the driver's driving style. The expert system module evaluates the driver's driving style and on that basis infers and issues tips. Below, we will describe each of the modules.

\section{A. Data adquistion Module}

As previously mentioned, the Artemisa's project aims to develop an eco-driving assistant that models the driving style and provides tips more accurately than other previous systems.

For this, the data acquisition system will get the value of all the variables that influence the energy consumption associated with both the car and the environment where it circulates.

Data acquisition systems, traditionally, only take into account the variables associated with the vehicle. In Table 1, a comparison between a traditional data acquisition and the proposed system is shown.

Another Artemisa's project objective is that the cost of the assistant is as cheap as possible, so we decided to use Android as a mobile data acquisition system as it is able to get lots of information affecting energy consumption without the need of installing additional hardware on board the vehicle. In addition it also uses a Bluetooth module which will obtain the data (speed, percentage of throttle down, gear, RPM, etc.) supplied by the vehicle's diagnostic port and sends them to the mobile device.

\begin{tabular}{|l|c|c|}
\hline & $\begin{array}{c}\text { Data acquisition } \\
\text { systems standards }\end{array}$ & $\begin{array}{c}\text { Artemisa's Data } \\
\text { acquisition } \\
\text { system }\end{array}$ \\
\hline Speed & Yes & Yes \\
\hline Acceleration & Yes & Yes \\
\hline RPM & Yes & Yes \\
\hline Gear & Yes & Yes \\
\hline Tire temperature & Yes & Yes \\
\hline Tire pressure & Yes & Yes \\
\hline Throttle position & Yes & Yes \\
\hline
\end{tabular}

\begin{tabular}{|l|c|c|}
\hline Brake position & Yes & Yes \\
\hline $\begin{array}{l}\text { Fuel } \\
\text { Consumption }\end{array}$ & Yes & Yes \\
\hline Model & Yes & Yes \\
\hline Slope Road & No & Yes \\
\hline Type Road & No & Yes \\
\hline State Road & No & Yes \\
\hline $\begin{array}{l}\text { Weather } \\
\text { conditions }\end{array}$ & No & Yes \\
\hline Location & No & Yes \\
\hline Speed (GPS) & No & Yes \\
\hline
\end{tabular}

Table 1. Comparison between a traditional data acquisition and the proposed system.

Below, we will describe the elements of the data acquisition system:

Bluetooth Adapter: Acts as interpreter converting diagnostic OBD2 port signals to serial data. Diagnostic port provides numerous information about engine control unit and other elements of the vehicle as the brake, TCM, ABS, etc.

To access this information we will use an identifier called PID. The SAE J1979 standard defines a basic list of PIDs and how to convert raw OBD-II diagnostic output to meaningful units but manufacturers are not required to implement all the PIDS and also include many no standard.

Android device: Execute Artemisa's eco-driving assistant (data acquisition system and expert system). Mobile device must have minimum characteristics to execute the assistant but this is not a problem since most of the devices on the market have these requirements. The features are:

GPS: It is used to determine the vehicle's position. If GPS signal is not available, use the network location. Know the location of the vehicle is required to get the weather of the environment and state road.

GPRS/3G/LTE Connectivity: Allow us to connect to the Internet to find out the state and weather conditions of the road. Web Services are used to obtain the information and provide the information in XML format. Android API supplies several methods (SAX, DOM and XMLPull) to parse XML and obtain the data that interest us.

Bluetooth connectivity: Allows to conect mobile device Bluetooth/OBD2 adapter to get the data supplied by the diagnostic port. Android's APIs from version 1.5 allows use socket communications using the Bluetooth interface from the mobile device so it must have this version or higher.

Light, Orientation and Accelerometer sensors: Allow to know the value of variables that influence in the consumed energy; in particular the slope road, the ambient light and the vehicle acceleration.

Light sensor allows us to determine the brightness of the environment where the vehicle is traveling. The brightness will significantly influence visibility in influencing the ability to anticipate the driver being is the key to efficient driving. The 
eco-driving assistant uses this information to alert the user to slow down if it is not suitable for environmental conditions.

Light sensor allows us to determine the brightness of the environment where the vehicle is traveling. The brightness will affect significantly the visibility influencing the capacity of anticipation of the driver being the key to efficient driving. The assistant will use this data to warn the user to decrease speed if it is not suitable for the conditions of the environment.

Orientation sensor determines if the vehicle is circulating uphill or downhill. Depending on the type of slope, eco-driving assistant will evaluate if the driver is performing actions appropriate from the point of view of energy saving. If the road is uphill, driver should try to maintain the gear. If the slope is downhill the driver should avoid press the brake pedal and the gear changes will be carried out in such a way that the tachometer needle is placed at the bottom of the green zone of the tachometer.

Acceleration sensor will allow us to find out when the driver accelerates, with what frequency and intensity with which it does. This information is very useful to evaluate the driver from the point of view of energy consumption. The acceleration can be obtained also through the vehicle's diagnostic port but it can happen that the manufacturer does not use a standard PID and therefore can not obtain the information.

On the other hand, sensors vehicles are subject to extreme conditions that cause a large decline in the same, we can use the sensors of the mobile device as back.

In figure 1 we can see Google Nexus S executing Artemisa's Data Acquisition System

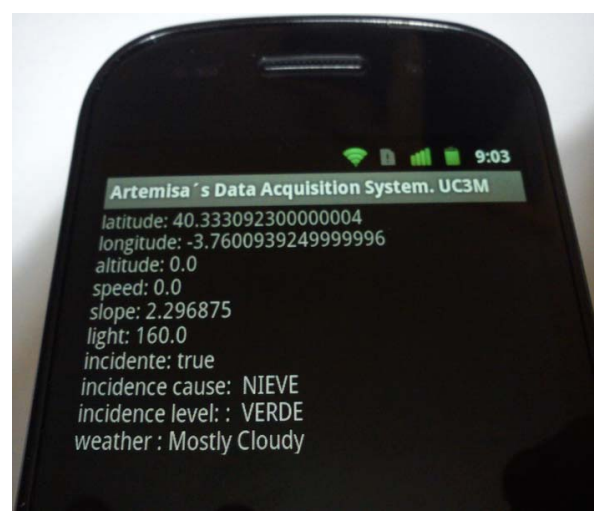

Figure 1. Google Nexus $\mathrm{S}$ executing Artemisa's Data Acquisition System

\section{B. Expert System}

Analyze the driving style from the point of view of energy consumption for then give eco-driving advice is a very complex problem due to the large number of variables involved. We propose a solution based on the use of an expert system that was executed on an Android device.

Current mobile devices have processors at $1 \mathrm{GHz}$ or even there are already devices with dual-core processors (TEGRA, Samsung Exynos and OMAP4) on the market. Also tend to have $512 \mathrm{MB}$ of RAM so they are powerful enough to execute complex tasks.

But due to the particularities of the underlying problem (issue eco-driving advice in real time based on the evaluation of the driving style) and to the platform above which will execute the solution, the classifier algorithm of the expert system must comply with the requirements that will be exposed below:

Speed: As eco-driving tips are displayed in real time is necessary for the classification algorithm is executed quickly. If the tips are issued later may not be valid. We also have to take into account that the classification model will have to rebuild every time when a new instance is classified because it will be stored in the knowledge base. The goal is to improve the system gradually.

Memory usage: Although current mobile devices have enough memory cannot compare to server or desktop computers. Classification algorithms tend to consume lots of memory, especially when the dataset is very large and this is the case in Artemisa project. Select a classification algorithm that consumes little memory is crucial to ensure that there will be no failures due to insufficient memory.

Handling instances with missing attribute values: Data acquisition system may not be able to provide the value of all the attributes used in the expert system. For example, if there is a disconnect Internet we could not get the state road or weather conditions. For this reason, it is necessary that the classification algorithm is able to handle instances with missing attribute values.

Artemisa's eco-driving assistant used Random Forest classification algorithm. The choice of this algorithm was made after a comparative study between the algorithms J48, Naive Bayes and Random Forest.

The algorithms were executed in a Nexus $\mathrm{S}$ with processor at $1 \mathrm{GHz}, 512 \mathrm{MB}$ of RAM and Android 2.3.1. For this study were used several datasets extracted from the UCI website [8] and the Weka library [9] adapted to Android.

Conclusions drawn were:

J48: Usually gets good results in classifying but consumes lots of memory and is the longer it takes to run.

Naïve Bayes: This algorithm is the simplest of the three and the most efficient in the use of mobile device resources. The drawback is that it is one of the less accurate algorithms, but presents good results in most of the time. This algorithm considers the attributes independent of each other and this is his major drawback.

Random Forest: It is more efficient than J48 and even its outcomes tend to be more precise. The drawback is that it is constructed to minimize the overall error rate and therefore it 
will tend to focus more on the prediction accuracy of the majority class, which often results in poor accuracy for the minority class. Even so it is rarely is overcome by $\mathrm{J} 48$.

\section{Expert System Structure}

Export System Module has four components:

- Preprocessing Module: It obtains more information using the data supplied by the data acquisition system. Furthermore, it deletes anomalous data.

- Facts Base: It stores the data obtained by the data acquisition system.

- Knowledge base: It contains the knowledge about eco-driving extracted from the manuals about efficient driving or experts.

- Classifier: It is responsible for generating eco-driving rules using the knowledge stored in the knowledge base.

Figure 2 shows a diagram of the expert system.

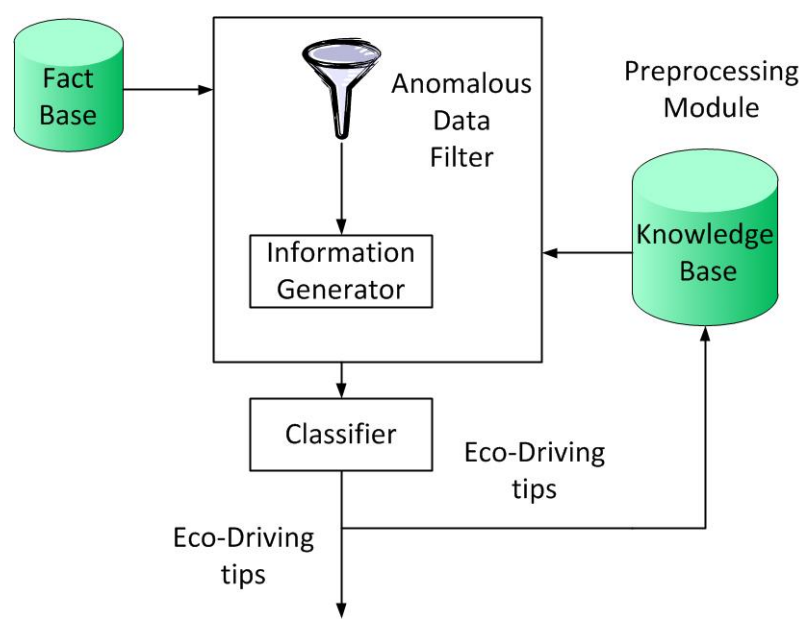

Figure 2. Expert System

Preprocessing module: are made up of two sub-modules:

- Filter of anomalous data: It has been observed that in some cases the data acquisition system gets anomalous values, for example when the vehicle is stopped the distance trip obtained from OBD2 port is unreal.

This sub-module is responsible for eliminating such data with the goal that the system does not degrade.
We use the data received earlier and will check if the new value of a variable obtained by the data acquisition system differs too from the previous values of that variable. Also an interval for each variable will be defined, if the value of the variable is not in the interval is discarded.

- Information Generator: It obtains more information using the data supplied by the data acquisition system. Example: OBD2 port provides the distance trip, if we observe that the distance trip has not changed over a period of time, we will know that the vehicle is found standing over that period of time. If that period is equal or higher than two minutes, the driver should turn off the engine to save fuel.

\section{Facts Base}

It is a database that stores the data obtained by the data acquisition system. Its format is:

ValueAttribute_1, ValueAttribute_2,..., ValueAttribute_i

The attributes are the set of variables (speed, gear, weather conditions, ect...) whose values are obtained by the data acquisition system. When the value of the attribute is not known we insert a question mark instead. Less recent information will remove to avoid which the database grows too.

\section{Knowledge Base}

It is a database that contains the knowledge (also called training set) extracted from the manuals about efficient driving [3] [4]. In addition, the knowledge base will increase with each new classified instance, in order to improve the response of the system in each new interaction.

However, the Expert System runs on a mobile platform with limited memory resources, so that Knowledge Base can not grow without control. Otherwise, if the knowledge base is too big, the construction of the classifier will consume too much time and the eco-driving tips may be issued too late.

To resolve this problem, we include new instances in the knowledge base while the build time of the classification model does not exceed a threshold.

In future work, we will explore a smart way to replace the instances introduced during the classification process for new ones, once the threshold has been exceeded. The aim is that the knowledge base contains instances that produce the best results.

The format of the instances stored in the knowledge base is as follows:

ValueAttribute_1,ValueAttribute_2,......, Class 
Attributes are the set of variables (speed, gear, weather conditions, etc....) whose values are obtained by the data acquisition system. When the value of the attribute is not known, we insert a question mark instead. Class represents an eco-driving advice. Below, we will show an example of the instance of the knowledge base.

$$
?, ?, ?, ?, ?, ?, ?, ?, ?, ?, ?, ?, 150, ?, ?, ?, ?, ?, 1
$$

This example expresses that if a vehicle circulates at 150 $\mathrm{km} / \mathrm{h}$ must be displayed the driving tip one. The advice one indicates that the user have to reduce speed because circulate at high speeds increases fuel consumption since is needed more power engine.

\section{CONCLUSION AND FUTURE WORK}

To model the driver's driving style in order to assess if he or she makes an efficient and optimized driving the only use of the data obtained through the vehicle diagnostic port (as many solutions of Eco-Driving incorporated in vehicles by the manufacturers do) is not enough. Other external information is required to put the system in context and therefore to obtain more precise influence on energy consumption.

The eco-driving assistant presented in this paper considers externals variables to the vehicle that significantly affect energy consumption. For doing this, we use the information that today's mobile smart devices allow us to get through its sensors and connectivity. The use of a Smartphone as ecodriving assistant also makes the solution cost low as compared to other solutions with specific hardware.

In future work, we will improve the assistant concentrating on the anticipation of the user because it is one of the most important factors in eco-driving. We will measure distances. For example, we can calculate the distance to the preceding vehicle and see if it is appropriate in order to avoid having to continually press the brakes (with the associated cost of energy). Also, we intend to measure the distance between where the vehicle is when the driver brakes and traffic signals, what force has to make it stop, to evaluate if the driver releases the accelerator in good time and applies the brakes as little as possible. Evaluate driver anticipation not only aims at saving fuel but it will also increase security. For measuring the distances the ARTEMISA's assistant will make use of the mobile device camera.

\section{ACKNOWLEDGEMENTS}

The research leading to these results has received funding by the ARTEMISA project TIN2009-14378-C02-02 within the Spanish "Plan Nacional de I+D+I", and the Madrid regional community projects S2009/TIC-1650 and CCG10UC3M/TIC-4992.

\section{REFERENCES}

[1] Spanish Society of Pneumology and Thoracic Surgery. URL: http://www.separ.es. January 2011.

[2] "Traffic: civilization or barbarism". The risk Observatory. Institute for security studies (IDES). URL: http://www.seguretat.org. 2006.

[3] IDEA (Institute for Energy Diversification and Saving of Energy). Manual of eco-driving for industrials vehicles. November 2005.

[4] IDEA (Institute for Energy Diversification and Saving of Energy). Manual of eco-driving for cars. January 2007.

[5] Jack N. Barkenbus, Eco-driving: An overlooked climate change initiative, Energy Policy, Volume 38, Issue 2, February 2010, Pages 762-769, ISSN 0301-4215, DOI: 10.1016/j.enpol.2009.10.021. (http://www.sciencedirect.com/science/article/B6V2W-4XJN4X45/2/6ffebae2ed6ed89c7f0c083fa7b37e6d).

[6] J.Van Mierlo , G.Maggetto, E.Van Burgwal, R Gense. "Driving style and traffic measures-influence on vehicle emissions and fuel consumption". Proceedings of the Institution of Mechanical Engineers, Part D: Journal of Automobile Engineering. Volume 218, Number $1 /$ 2004. PP: 43-501

[7] Kanok Boriboonsomsin, Alexander Vu, and Matthew Bart. "Co EcoDriving: Pilot Evaluation of Driving Behavior Changes among U.S. Drivers". University of California Transportation Center. 2009

[8] UCI. Url: http://archive.ics.uci.edu/ml/. April 2011

[9] Weka for Android. Url: https://github.com/rjmarsan/Weka-for-Android. April 2011

[10] Android. Url: http://developer.android.com/index.html. April 2011.

[11] Kanok Boriboonsomsin, Alexander Vu, and Matthew Bart. "Co EcoDriving: Pi-lot Evaluation of Driving Behavior Changes among U.S. Drivers". University of California Transportation Center. 2009

[12] J.Van Mierlo , G.Maggetto, E.Van Burgwal, R Gense. "Driving style and traffic measures-influence on vehicle emissions and fuel consumption". Proceedings of the Institution of Mechanical Engineers, Part D: Journal of Automobile Engineering. Volume 218, Number 1 2004. PP: 43-501

[13] Kuhler, M., Kartens, D., "Improved driving cycle for testing automotive exhaust emissions". SAE Technical Paper Series 780650. 1978

[14] André, M., 1996. Driving cycles develop-ment: Characterization of the methods. SAE Technical Papers Series 961112

[15] Fomunung, I., Washington, S., Guensler, R. "A statistical model for estimating oxides emissions from light duty motor vehicles". Transporta-tion Research Part D, pp.333-352. 1999 